\title{
Valoración de las Cargas en la Cosechadora de Caña de Azúcar en las Condiciones de Cuba
}

\author{
Ing. Juan Rafael Perez Pupo, PhD \\ Escuela Superior Politécnica de Chimborazo (ESPOCH), \\ Facultad de Mecánica, Escuela de Ingeniería Industrial, Ecuador \\ Ing. Julián Remberto Sánchez Alonso, PhD \\ Universidad de Holguín (UHO), Facultad de Mecánica, Cuba \\ Ing. Julio Nolberto Pérez Guerrero, PhD \\ Universidad Técnica de Manabí (UTM) Facultad de Ciencias Matemáticas, \\ Físicas y Químicas, Ecuador \\ Ing. Carlos José Santillán Mariño, MsC \\ Escuela Superior Politécnica de Chimborazo (ESPOCH), \\ Facultad de Mecánica, Escuela de Ingeniería Industrial, Ecuador
}

Doi:10.19044/esj.2019.v15n21p294 URL:http://dx.doi.org/10.19044/esj.2019.v15n21p294

\section{Resumen}

Las cargas de explotación en las cosechadoras de caña han sido insuficientemente estudiadas y constituyen la base del modelo estadístico de las cargas de explotación como información de entrada al sistema dinámico. El experimento se llevó a cabo en un diseño con un esquema factorial ( 3 x 2), utilizando tres rendimientos del cultivo y dos sentidos de inclinación de las plantas, con tres repeticiones. En el trabajo se utilizó la extensometría como método de investigación experimental y con ayuda de la estadística descriptiva y dinámica se obtuvieron los parámetros estadísticos de las cargas de explotación de la cosechadora de caña para diferentes regímenes y condiciones de explotación del este de Cuba, así como las características dinámicas de las mismas. Como resultado del trabajo se constató que el consumo de potencia para la realización del proceso tecnológico es del $84 \%$, los órganos con mayor consumo de potencia son los discos de corte base $(20.4 \mathrm{~kW})$ y el aparato trozador $(11.8 \mathrm{~kW})$. El espectro de potencia del momento torsor en el aparato trozador oscila en el rango de 0 a $12 \mathrm{~Hz}$, la eficiencia del sistema hidráulico fue del $61 \%$. La zona de baja frecuencia se produce por la acción de la masa cosechada y especialmente el flujo de caña a lo largo del surco. Se demostró en condiciones de campo el hecho de que el consumo de potencia en el árbol superior del aparato trozador es de un $30 \%$ superior a la consumida por el tambor inferior. Esta información es la base para el proceso de desarrollo de estos sistemas mecánicos. 
Palabras clave: Caña de azúcar, Cosechadora, Mecanismo trozador, Cargas de explotación, Potencia

\title{
Assessment of the Loads in the Sugar Cane Harvester Under the Conditions of Cuba
}

\author{
Ing. Juan Rafael Perez Pupo, PhD
}

Escuela Superior Politécnica de Chimborazo (ESPOCH), Facultad de Mecánica, Escuela de Ingeniería Industrial, Ecuador

Ing. Julián Remberto Sánchez Alonso, PhD

Universidad de Holguín (UHO), Facultad de Mecánica, Cuba

Ing. Julio Nolberto Pérez Guerrero, PhD

Universidad Técnica de Manabí (UTM) Facultad de Ciencias Matemáticas,

Físicas y Químicas, Ecuador

Ing. Carlos José Santillán Mariño, MsC

Escuela Superior Politécnica de Chimborazo (ESPOCH), Facultad de Mecánica, Escuela de Ingeniería Industrial, Ecuador

\begin{abstract}
The operation loads in the sugar cane harvesters have been insufficiently studied and they constitute the base of the statistical model of the operation loads like entrance information to the dynamic system. The experiment was carried out in a design with a factorial scheme $(3 \times 2)$, using three crop yields and two directions of inclination of the plants, with three repetitions. In the work making use of the electric extensometry as experimental research method and with the help of the descriptive statistic and dynamics the statistical parameters of the operation loads were obtained in the sugar cane harvester for regimen different and operation conditions of eastern Cuba were obtained, as well as the dynamic characteristics of the same ones. As a result of the work it was found that the power consumption for the realization of the technological process is $84 \%$, the organs with the highest power consumption are the base cutting discs $(20.4 \mathrm{~kW})$ and the cutting unit $(11.8 \mathrm{~kW})$. The power spectrum of the torque in the splitter oscillates in the range of 0 to $12 \mathrm{~Hz}$, the efficiency of the hydraulic system was $61 \%$. The zone of low frequency is produced by the action of the harvested mass and especially the flow of cane along the furrow. It was demonstrated under field
\end{abstract}


conditions that the power consumption in the upper tree of the splitter apparatus is $30 \%$ higher than that consumed by the lower drum. This information is the basis for the development process of these mechanical systems.

Keywords: Sugar cane, Sugar cane harvester, Crosscutter device, Operation loads, Power

\section{Introducción}

La creación de máquinas de alto rendimiento y confiables para cosechar la caña de azúcar es actualmente el punto más importante para aumentar la producción y reducir el costo de un producto tan importante para Cuba como el azúcar.

Sin embargo, el desarrollo de un diseño óptimo de una cosechadora requiere una gran cantidad de trabajo, no solo para mejorar el funcionamiento de los órganos de trabajo, sino también para aumentar la confiabilidad y al mismo tiempo reducir el peso total de la máquina. Cabe señalar que el coeficiente de disponibilidad técnica de las cosechadoras de caña que se producen actualmente en el país es menor y que el índice de consumo de metal con respecto a la productividad es mayor que las de las comercializadas mundialmente y, por lo tanto, mejorar la calidad de las máquinas de nuevo diseño es una tarea urgente. El deseo de crear una máquina con determinados parámetros de calidad requiere, sobre todo, la disponibilidad de datos básicos sobre las cargas de cada órgano de trabajo et. al. 2015). La ausencia de dicha información sobre las cosechadoras de caña ha requerido la realización de trabajos de investigación científica sobre las cargas operativas de los órganos de trabajo bajo la influencia de diferentes condiciones del material a cosechar (variedades, rendimientos, edades de las plantaciones etc.) a cosechar y del medio (microrelieve de los campos, tipos de suelo y condiciones climáticas) donde se explotan en Cuba. Este trabajo fue llevado a cabo por la Universidad de Holguín junto con el Centro para el Desarrollo de la Maquinaria Agrícola (CEDEMA) sobre un prototipo de cosechadora de caña con accionamiento hidráulico en la totalidad de sus mecanismos.

Los estudios sobre las cargas operativas (de funcionamiento) de varias máquinas móviles han demostrado que se pueden obtener características fiables solo teniendo en cuenta el conjunto completo de condiciones de carga, la naturaleza del relieve del suelo, la velocidad de movimiento de la cosechadora, la realización del proceso tecnológico o el movimiento de transporte, el rendimiento de los cultivos cosechados, etc. (Davis, Whiteing, \& Norris, 2010; Ma, Karkee, Scharf, \& Zhang, 2014; Peloia, Milan, \& Romanelli, 2010, Faheem, et. al. 2016, Ramos, C. R, et. al. 2016). 
En correspondencia con lo antes consignado el trabajo tuvo por objetivo caracterizar estadísticamente las cargas de explotación bajo diferentes condiciones naturales de operación de los principales órganos y sistemas de trabajo de la cosechadora de caña con el propósito de obtener información de valor para los trabajos de desarrollo de esta importante máquina para la economía de Cuba.

\section{Materiales y métodos}

El experimento se llevó a cabo en un diseño con un esquema factorial ( 3 x 2), utilizando tres rendimientos del cultivo y dos sentidos de inclinación de las plantas, con tres repeticiones.

El rendimiento tiene tres niveles: alto, medio y bajo para una misma variedad y la inclinación dos: a favor y en contra. El diseño experimental se muestra en la tabla 1.

Tabla 1. Diseño Experimental.

\begin{tabular}{|l|l|l|l|}
\hline \multirow{2}{*}{ Inclinación de las plantas } & \multicolumn{3}{|c|}{ Rendimiento } \\
\cline { 2 - 4 } & Alto & Medio & Bajo \\
\hline A favor & & & \\
\hline En contra & & & \\
\hline
\end{tabular}

Para cumplir los objetivos propuestos las pruebas se realizaron en el régimen de cosecha durante la realización del proceso tecnológico. El ensayo de cosecha se divide en dos partes: vacío y cosecha.

Las mediciones tensométricas fueron realizadas por un colectivo multidisciplinario a un prototipo de cosechadora de caña con el $100 \%$ de sus mecanismos accionados hidráulicamente. A la cosechadora se le realizaron las pruebas en la provincia de Holguín, al Oriente del país. En las pruebas se midieron los siguientes parámetros (Fig. 1): momento torsor y revoluciones por minuto en el lado derecho del mecanismo de corte inferior, momento torsor y revoluciones en el aparato trozador, revoluciones en las ruedas motrices y revoluciones de la quinta rueda de la cosechadora.

Las mediciones se realizaron según la metodología utilizada, las pruebas en cosecha se efectuaron en dos variedades de caña comerciales (Instituto Nacional de Investigaciones de la caña de azúcar (INICA), 2010, Sureshkumar P.K., \& Manohar Jesudas D. 2015). Estas se llevaron a cabo con cuatro variedades de caña con rendimiento que oscilaron entre 55 a $200 \mathrm{t} / \mathrm{ha}$. el tiempo de medición de cada registro osciló entre 120 a $150 \mathrm{~s}$, lo cual se fundamenta en que el límite superior considerado de las frecuencias del proceso es de hasta $30 \mathrm{~Hz}$, con una discretización de las señales en pasos de $0,01 \mathrm{~s}$, con lo cual se dispuso del número de registros necesarios. se realizaron mediciones de las características de las plantaciones a saber rendimiento, flujo tecnológico, diámetro y altura de las plantas etc. 
Para la realización de las pruebas se empleó el equipamiento tensométrico móvil del CEDEMA. Para el almacenamiento y procesamiento de la información se utilizó una computadora personal acoplada al sistema de medición (Chatfield, Bendat, \& Piersol, 2006; Lurie, 1970; Sharpe, 2008, Sureshkumar P.K., \& Manohar Jesudas D. 2015, Wang, F., et. al. 2018).

Para la medición del momento torsor en los árboles se empleó el método activo, mediante la fijación directa de las galgas en el árbol, las cuales se instalaron según el esquema del semipuente de cuatro captadores activos (Sharpe, 2008).

Para determinar las velocidades angulares de los árboles y la velocidad de traslación de las máquinas se instalaron captadores inductivos y tacogeneradores.

El tiempo de trabajo de las cosechadoras de caña en cada modo típico de operación se determinó a partir de las fotografías de la jornada laboral realizada por la Universidad de Holguín.

El procesamiento estadístico, una vez eliminados los tramos transitorios no estacionarios, se realizó según los métodos determinístico y dinámico de la estadística. El análisis determinístico, se efectuó a partir de la base de datos general, obtenida para todos los regímenes de explotación investigados, con lo cual se obtuvieron las distribuciones sumarias de cada parámetro estudiado y con ello su caracterización estadística general a sabermedia, rango, varianza etc.

El estudio dinámico se efectuó a partir del conjunto de realizaciones para los tramos estacionarios asumiendo que el proceso es estacionario y ergódico, con lo cual, empleando la teoría de procesos estocástico, se determinó la función correlativa y la densidad espectral.

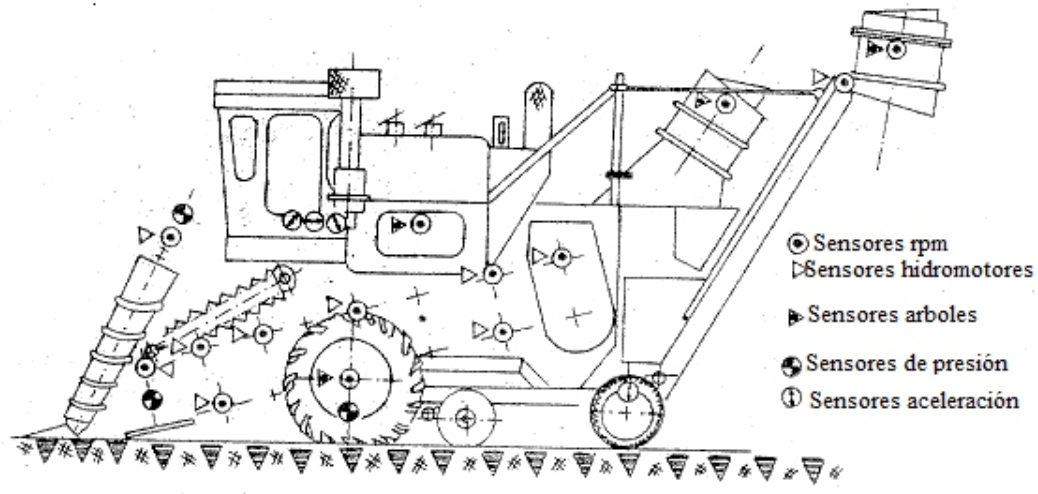

Figura 1. Disposición de los elementos dinamométricos en la cosechadora de caña

\section{Resultados y discusión}

De acuerdo con los valores sumarios, los modos de operación de las cosechadoras para cosechar la caña de azúcar durante la operación en las 
provincias del este de Cuba ascendieron a 0,7 para el trabajo, y 0,3 para el transporte del total de tiempo de funcionamiento. La aparición de diferentes condiciones dentro de cada modo se considera igualmente probable.

Como resultado de las pruebas de campo, se formó un banco de datos que contenía 4,2E06 valores discretos. El procesamiento estadístico de los resultados de la investigación se llevó a cabo con la ayuda de computadoras personales con ayuda de los programas estadísticos clásicos y programas originales desarrollados en la Universidad de Holguín. El propósito del procesamiento del material experimental fue la obtención de una característica de carga de las distribuciones sumarias de las cargas de cada órgano de trabajo de la cosechadora (Tabla 2), incluida la carga para 12 modos de trabajo y 4 de transporte.

Tabla 2. Características de carga sumaria de los órganos de trabajo para la cosecha de caña de azúcar.

\begin{tabular}{|c|c|c|c|c|c|c|c|c|c|c|c|}
\hline \multirow[t]{3}{*}{$\mathrm{N}^{\circ}$} & \multirow[t]{3}{*}{ Punto de medición } & \multicolumn{7}{|c|}{ Vacío } & \multicolumn{3}{|c|}{ Trabajo } \\
\hline & & $\mathrm{T}$ & Tmax. & $\mathrm{n}$ & $\mathrm{N}$ & Nmax. & $\mathrm{T}$ & Tmax & $\mathrm{n}$ & $\mathrm{N}$ & Nmax. \\
\hline & & $\mathrm{Nm}$ & $\mathrm{Nm}$ & rpm & $\mathrm{kW}$ & $\mathrm{kW}$ & $\mathrm{Nm}$ & $\mathrm{Nm}$ & $\mathrm{rpm}$ & $\mathrm{kW}$ & $\mathrm{kW}$ \\
\hline 1 & $\begin{array}{|llll|}\text { Cardan } & \text { a la } & \text { salida del } \\
\text { motor } & \text { de } & \text { combustión } \\
\text { interna } & & \\
\end{array}$ & 351 & 420 & 2296 & 84,39 & 101,5 & 470 & 781 & 2235 & 110 & 182,79 \\
\hline 2 & $\begin{array}{|ll|}\begin{array}{l}\text { Trozador lado izquierdo } \\
\text { (inferior) }\end{array} & \\
\end{array}$ & 17 & 55 & 331 & 0,59 & 1,91 & 30 & 307 & 300 & 0,94 & 9,64 \\
\hline 3 & $\begin{array}{|lll|}\begin{array}{l}\text { Trozador } \\
\text { (superior) }\end{array} & \text { lado } & \text { derecho } \\
\end{array}$ & 10 & 28 & 331 & 0,35 & 0,97 & 56 & 449 & 300 & 1,76 & 14,11 \\
\hline 4 & Trozador sumario & & & 331 & 0,94 & 2,88 & & & 300 & 2,7 & 23,75 \\
\hline 5 & Disco de corte base & 17 & 37 & 648 & 2,51 & 2,51 & 69 & 347 & 605 & 4,37 & 21,98 \\
\hline 6 & I Extractor & 13,6 & 32 & 858 & 2,88 & 2,88 & 24,6 & 39,2 & 905 & 2,33 & 3,72 \\
\hline 7 & I Tambor lado izquierdo & 39 & 91 & 93 & 0,89 & 0,89 & 7676 & 239 & 99 & 0,79 & 2,48 \\
\hline 8 & I Tambor lado derecho & 32 & 89 & 93 & 0,87 & 0,87 & 4444 & 151 & 99 & 0,46 & 1,57 \\
\hline 9 & I Tambor sumario & & & 93 & 0,00 & 0,00 & & & 99 & 1,25 & 4,05 \\
\hline 10 & III Tambor & 2,2 & 12,4 & 294 & 0,38 & 0,38 & 20 & 96 & 287 & 0,60 & 2,89 \\
\hline 11 & IV Tambor & 2,6 & 22,5 & 273 & 0,64 & 0,64 & 31 & 134 & 286 & 0,93 & 4,01 \\
\hline 12 & VI Tambor & 3,8 & 31 & 290 & 0,94 & 0,94 & 125 & 164 & 287 & 3,76 & 4,93 \\
\hline 13 & VII Tambor lado izquierdo & 3.0 & 14 & 262 & 0,08 & 0,38 & 14 & 54 & 245 & 0,36 & 1,39 \\
\hline 14 & VII Tambor lado derecho & 10,0 & 22 & 262 & 0,27 & 0,60 & 30 & 108 & 245 & 0,77 & 2,77 \\
\hline 15 & VII Tambor sumario & & & 262 & 0,35 & 0,98 & & & 245 & 1,13 & 4,16 \\
\hline 16 & II Tambor & 2,0 & 5,38 & 281 & 0,06 & 0,16 & 6 & 79 & 285 & 0,18 & 2,36 \\
\hline 17 & Sinfín & 2,0 & 22,4 & 298 & 0,06 & 0,70 & 18,6 & 166 & 289 & 0,56 & 5,02 \\
\hline 18 & II Extractor & 27,5 & 59,5 & 808 & 2,33 & 5,03 & 33 & 78,6 & 909 & 3,14 & 7,48 \\
\hline 19 & $\begin{array}{l}\text { Transportador de descarga } \\
\text { lado izquierdo }\end{array}$ & 7,8 & 16 & 268 & 0,22 & 0,45 & 12,5 & 27 & 256 & 0,34 & 0,72 \\
\hline 20 & $\begin{array}{l}\text { Transportador de descarga } \\
\text { lado derecho }\end{array}$ & 12,4 & 25,8 & 268 & 0,35 & 0,72 & 26,7 & 56 & 255 & 0,71 & 1,50 \\
\hline
\end{tabular}




\begin{tabular}{|l|l|c|c|c|c|c|c|c|c|c|c|}
\hline 21 & $\begin{array}{l}\text { Transportador de descarga } \\
\text { lado sumario }\end{array}$ & & 268 & 0,57 & 1,17 & & & 255 & 1,05 & 2,22 \\
\hline 22 & Rueda motriz izquierda & 3457 & 5647 & 3,04 & 1,10 & 1,80 & 4136 & 10548 & 6,48 & 2,81 & 7,16 \\
\hline 23 & Rueda motriz derecha & 3589 & 5684 & 3,04 & 1,14 & 1,81 & 4488 & 11428 & 6,48 & 3,05 & 7,75 \\
\hline 24 & Puente motriz sumario & & & 3,04 & 2,24 & 3,61 & $\mathrm{~V}=2,34 \mathrm{~km} / \mathrm{h}$ & 6,48 & 5,86 & 14,91 \\
\hline
\end{tabular}

Los valores de los parámetros estudiados representan los más probables durante la explotación y su rango de variación, lo cual es una información de mucho valor para los cálculos ingenieros en condiciones estáticas. el análisis de la tabla muestra que el consumo de potencia para la realización del proceso tecnológico es del $84 \%$, los órganos con mayor consumo de potencia son los discos de corte base $(20.4 \mathrm{~kW})$ y el aparato trozador $(11.8 \mathrm{~kW})$. El espectro de potencia del momento torsor en el aparato trozador oscila en el rango de 0 a $12 \mathrm{~Hz}$, la eficiencia del sistema hidráulico fue del $61 \%$. La zona de baja frecuencia se produce por la acción de la masa cosechada y especialmente el flujo de caña a lo largo del surco. Se demostró en condiciones de campo el hecho de que el consumo de potencia en el árbol superior del aparato trozador es de un $30 \%$ superior a la consumida por el tambor inferior

Según el análisis de los datos en el modo de trabajo, el flujo de potencia se distribuye de la siguiente manera:

$\checkmark$ Proceso tecnológico - $84 \%$

$\checkmark$ Desplazamiento de la cosechadora $-5,5 \%$

$\checkmark$ Equipo auxiliar $-10,5 \%$

La velocidad de movimiento de la cosechadora varía según el rendimiento del cultivo cosechado. Con un aumento en el rendimiento, la velocidad de movimiento de la cosechadora disminuye bruscamente, y el par de torsión en las ruedas motrices se mantiene. En los modos de transporte, la velocidad de la cosechadora se rige por la calidad de los caminos de campo y la condición cómoda del conductor.

Al procesar los datos, se observó que los tramos de naturaleza no estacionaria de los procesos de acuerdo con la esperanza matemática disminuyen considerablemente con el aumento de la cantidad de datos que se procesan. En la práctica, estos registros desaparecieron con tamaños de muestra de 3000 a 3500 valores. Este fenómeno indica la presencia en el proceso de un componente "lento", que difiere de la frecuencia dominante en 1-2 órdenes de magnitud.

La función correlativa elaborada a partir del análisis dinámico de las señales se muestra para el órgano trazador (Fig. 2). El espectro de potencia del momento torsor oscila en el rango de 0 a $12 \mathrm{~Hz}$, dentro de este hay una zona de alta frecuencia entre los 5 y los $12 \mathrm{~Hz}$ que se corresponde con el doble de 
la frecuencia de rotación del aparato trozador durante su trabajo (debido a los dos cortes que se producen en cada vuelta del tambor). La zona de baja frecuencia se produce por la acción de la masa cosechada y especialmente el flujo de caña a lo largo del surco.

De hecho, la combinación compleja de oscilaciones de las frecuencias naturales en las frecuencias más bajas en combinación con las irregularidades de la masa del producto tecnológico que ingresa a la cosechadora provoca una naturaleza estocástica compleja de la variación del momento torsor en los órganos de trabajo.

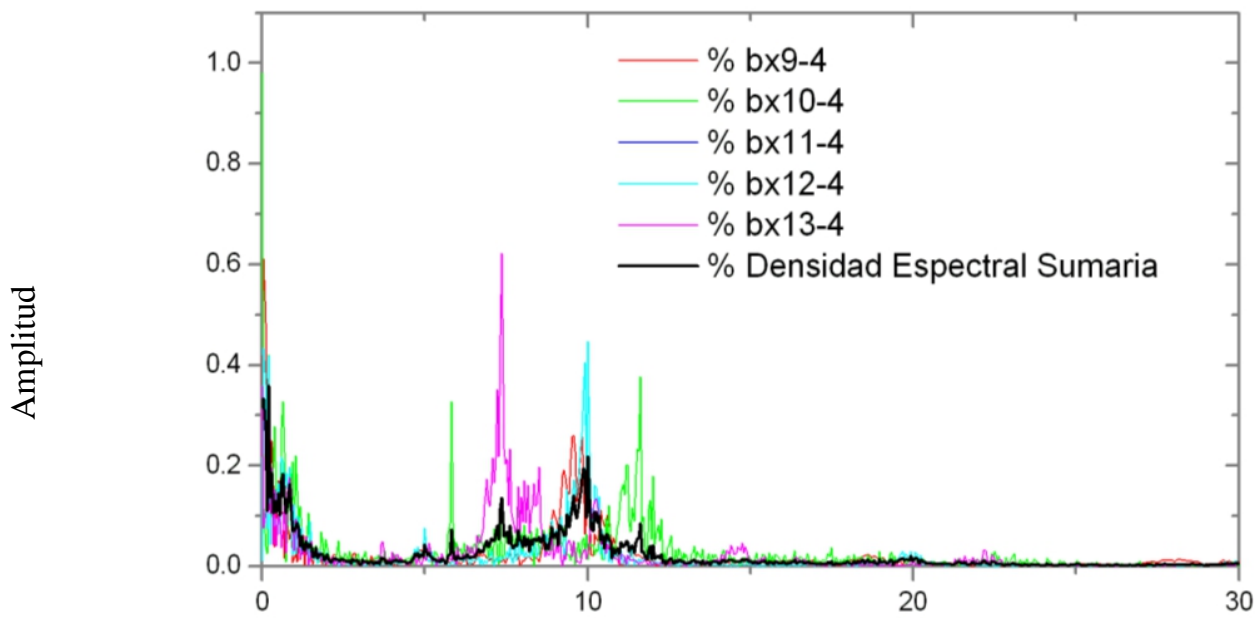

Figura 2. Densidad espectral del torque en el aparato trozador de la cosechadora de caña

\section{Conclusiones}

1. Se caracterizaron estadísticamente las cargas de explotación bajo diferentes condiciones naturales de operación de los principales órganos y sistemas de trabajo de la cosechadora de caña.

2. En el modo de trabajo, el flujo de potencia se distribuye de la siguiente manera:

$\checkmark$ Proceso tecnológico $-84 \%$

$\checkmark$ Desplazamiento de la cosechadora $-5,5 \%$

$\checkmark$ Equipo auxiliar $-10,5 \%$

3. Los órganos con mayor consumo de potencia son los discos de corte base $(20.4 \mathrm{~kW})$ y el trozador $(11.8 \mathrm{~kW})$.

4. El 39\% de la energía requerida para el flujo de trabajo se pierde debido a la eficiencia del accionamiento hidráulico.

\section{References:}

1. Chatfield, C., Bendat, J. S., \& Piersol, A. G. (2006). Random Data: Analysis and Measurement Procedures. In Journal of the Royal Statistical Society. Series A (General) (Vol. 150). 
https://doi.org/10.2307/2981634

2. Cox, G. J. (2002). A yield mapping system for sugar cane chopper harvesters. Retrieved from https://eprints.usq.edu.au/4617/

3. Davis, R., Whiteing, C., \& Norris, C. (2010). A review of opportunities to improve the design and performance of sugarcane harvesters. Retrieved from https://elibrary.sugarresearch.com.au/handle/11079/12632

4. Faheem, Tanzeel ur Rehman Muhammad Usman Khan Muhammad Tayyab Muhammad Waqar Akram Muhammad. (2016). Current status and overview of farm mechanization in Pakistan $-\mathrm{A}$ review. AgricEngInt: CIGR Journal, 18(2), 83-93.

5. Instituto Nacional de Investigaciones de la Caña de Azúcar (INICA). (2010). Principales variedades de Caña de Azúcar propagadas en Cuba al cierre del 2008. ATAC, (1), 19-25.

6. Jena, P. P., Mahapatra, N., Khandai, S., \& Behera, D. (2018). Studies on the Operational Parameters of Sugarcane Harvesting Blades. International Journal of Current Microbiology and Applied Sciences, 7(03), 1163-1172. https://doi.org/10.20546/ijcmas.2018.703.138

7. Kishore, N., Gayathri, D., Venkatesh, J., Rajeswari, V., Sangeeta, B., \& Andchandrika, A. (2017). Present mechanization status in sugarcane -A Review. International Journal of Agriculture Sciences, 9(22), 4247-4253.

8. Lurie, A. B. (1970). Dinámica Estadística de los agregados agrícolas. Leningrado: Editorial Kolos.

9. Ma, S., Karkee, M., Scharf, P. A., \& Zhang, Q. (2014). Sugarcane Harvester Technology: A Critical Overview. Applied Engineering in Agriculture, 30(5), 727-739. https://doi.org/10.13031/aea.30.10696

10. Mathanker, S. K., Gan, H., Buss, J. C., Lawson, B., Hansen, A. C., \& Ting, K. C. (2015). Power requirements and field performance in harvesting energycane and sugarcane. Biomass and Bioenergy, 75, 227-234. https://doi.org/10.1016/J.BIOMBIOE.2015.02.025

11. Najafi, P., Asoodar, M. A., Marzban, \& Ahormozi, M. (2015). Reliability evaluation and analysis of sugarcane 7000 series harvesters in sugarcane harvesting. journal of agricultural machinery, 5(2), 446455.

Retrieved

from https://www.sid.ir/en/journal/ViewPaper.aspx?id=546791

12. Peloia, P. R., Milan, M., \& Romanelli, T. L. (2010). Capacity of the mechanical harvesting process of sugar cane billets. Scientia Agricola, 67(6), 619-623. https://doi.org/10.1590/S0103-90162010000600001

13. Pogorelyı̆, L. V. (Leonid V. (1991). Métodos de ingeniería de prueba para máquinas agrícolas. Retrieved from https://search.rsl.ru/ru/record/01001582884 
14. Ramos, C. R. G., Lanças, K. P., Lyra, G. A. de, Sandi, J., Ramos, C. R. G., Lanças, K. P., ... Sandi, J. (2016). Fuel consumption of a sugarcane harvester in different operational settings. Revista Brasileira de Engenharia Agrícola e Ambiental, 20(6), 588-592. https://doi.org/10.1590/18071929/agriambi.v20n6p588-592

15. Sharpe, W. N. (2008). Springer handbook of experimental solid mechanics.

Retrieved

from https://books.google.com.ec/books?id=I_1y1OkqZqQC\&dq=strain+g age+handbook\&hl=es\&source=gbs_navlinks_s

16. Sureshkumar P.K., \& Manohar Jesudas D. (2015). Physicomechanical properties of sugar cane stalks related to mechanical harvesting. Journal of Tropical Agriculture., 53(1). Retrieved from http://jtropag.kau.in/index.php/ojs2/article/view/330

17. Wang, F., Yang, G., Ke, W., \& Ma, S. (2018). Effect of Sugarcane Chopper Harvester Extractor Parameters on Impurity Removal and Cane Losses. IFAC-PapersOnLine, 51(17), 292-297. https://doi.org/10.1016/J.IFACOL.2018.08.178 